\title{
Rituximab for granulomatous lymphocytic interstitial lung disease in a patient with common variable immunodeficiency. Is single therapy enough?
}

\begin{abstract}
Granulomatous Lymphocytic Interstitial Lung Disease (GLILD) is an inflammatory pulmonary complication of common variable immunodeficiency (CVID) with distinctive patterns in the biopsy; Granulomatous Disease, Lymphocytic Interstitial Disease, Follicular Bronchiolitis and Organizing Pneumonitis. Without the proper treatment this complication will lead to important pulmonary dysfunction due to fibrosis. Although this process has been well recognized, protocols for a standardized treatment, and the proper timing for the use of immune modulation as well as the maintenance time with monoclonal antibodies are an unclear topic.
\end{abstract}

Case presentation: We present the case of a 57-year-old female patient with CVID. During the evaluation of an episode of autoimmune hemolytic anemia, she presented concomitantly radiological and histopathological features of GLILD. She was treated successfully with Rituximab monotherapy without any complications and is currently asymptomatic.

Conclusion: We conclude that the use of Rituximab as single therapy, modulates the lymphocytic infiltration of the lung parenchyma and stops the progression and organization of the damage mediated by $B$ cells and indirectly by T cells, and it could be used as monotherapy in the proper timing of the diagnostic process.

Keywords: common variable immunodeficiency $\cdot$ granulomatous lymphocytic interstitial lung disease • autoimmune hemolytic anemia $\bullet$ idiopathic thrombocytopenic purpura $•$ pulmonary function tests

\section{Introduction}

Granulomatous- Lymphocytic Interstitial Lung Disease (GLILD) is an inflammatory pulmonary complication of common variable immunodeficiency (CVID) with distinctive patterns in the biopsy; granulomatous disease, lymphocytic interstitial disease, follicular bronchiolitis and areas of organizing pneumonia [1,2]. The immunological data of the pathophysiology suggests that it is initiated by an infiltration of $\mathrm{T}$ as well as $\mathrm{B}$ lymphocytes and macrophages, that will further lead the progression of the inflammatory process to fibrosis [3,4]. Regarding the treatment of this disease there are many immunomodulatory treatments with few standardized protocols, but recent studies $[5,6]$ suggests that the combination of Rituximab and Azathioprine could be effective for preserving the pulmonary function. We present the case of a CVID patient with no circulating B lymphocytes in peripheral blood, which developed GLILD without any symptoms and with normal pulmonary function tests. The patient showed an important improvement of the pulmonary infiltration in chest X-ray after the use of single therapy with rituximab and currently is asymptomatic with no pulmonary complications.

\section{Case presentation}

A 57-year-old Caucasian woman with CVID seen by the immunology team since 1999 for the following immunological alterations: IgG $245 \mathrm{mg} / \mathrm{dL}, \operatorname{IgM} 31 \mathrm{mg} / \mathrm{dL}, \operatorname{IgA} 11 \mathrm{~g} / \mathrm{dL}, \mathrm{IgG}$ antibodies to tetanus and to pneumococcus undetectable with poor response after vaccination, and peripheral $\mathrm{B}$ lymphocytes of less than 1\%, CD19+CD27+IgM-IgD- B-cells: $0.7 \%$.

No history of recurrent infections was present at the time. She was tonsillectomized at 8 years old, and hysterectomized at 30 years old for

\section{Mauricio Arraya*1, Yolanda Castro', Joaquin Navarro', Elizabeth Sarmiento', Eduardo Fernández-Cruz' \& Javier Carbone Campoverde ${ }^{1}$ \\ 'Group of Clinical Immunology, Gregorio Maranon University General Hospital, Madrid, Spain \\ 2Pathological Anatomy Service, Gregorio Maranon University General Hospital, Madrid, Spain \\ *Author for correspondence: mac777met369@hotmail.com}


presenting recurrent metrorrhagia and multiple uterine fibroadenomas. One of her sisters was diagnosed of CVID with presentation of autoimmune cytopenias (AIHA).

From 1981 to 2000 she suffered four episodes of Idiopathic Thrombocytopenic Purpura (ITP), manifested as multiple hematomas with severe thrombocytopenia getting to a minimum of 12,000 platelets $/ \mu \mathrm{L}$ in the most severe episode. The immunological study showed negative antiplatelet antibodies. Every ITP relapse was treated with 30 to $60 \mathrm{mg}$ of Prednisone with good response to the treatment.

From 2001 to the year 2009 the profile of her clinical manifestation changed to purely red blood cells alterations with a normal account of platelets. The patient started feeling more tired and headaches were present more often. During the most intense episode of asthenia, the patient was hospitalized. The lab results showed hemoglobin of $6.5 \mathrm{~g} / \mathrm{dL}$, hematocrit of $19.5 \%$, and a positive direct Coombs test. Autoantibody profile of the patient included: anti-thyroid antibodies positive, antinuclear antibodies negative, antiphospholipid antibodies negative. High doses of Intravenous Immunoglobulins $(1 \mathrm{gr} / \mathrm{Kg} /$ day) and corticosteroid therapy were started. A neck, chest, and abdominal CT scan was performed, showing the presence of ground glass image in the left lower lobe, right lower lobe and right middle lobe with multiple adenopathies in the retroperitoneal space and mild Splenomegaly. Figure 1A the pulmonary alterations were not associated with any clinical manifestations.

The patient presented two more episodes of AHA in the years 2003 and 2005 respectively, treated successfully with the steroids and IVIG. In the year 2005 a Chest CT scan was performed to make a follow up of the pulmonary lesions. In this study, there were no changes in the pulmonary lesions showing the same ground glass images as before in the year 2001 Figure 1B.

Although she didn't have any pulmonary symptoms, and the pulmonary function tests were normal, a video assisted thoracoscopy (VATS) biopsy (left lower lobe) was performed showing a granulomatous infiltration with the presence of CD4 and CD8 T-Lymphocytes Figure 2A-D, and CD19/CD20 lymphocytes Figure 3A and B. $B$ lymphocytes were found predominantly and were organized in isolated follicular like structure surrounding the granulomas. The diagnosis of GLILD granulomatous pattern and organizing pneumonitis was established.

During this study a functional immunological study of peripheral blood was performed showing the following results Table 1 .

In the year 2008 the patient presented a new episode of AIHA, which was refractory to high dose of IVIG and corticosteroid therapy. Hence Rituximab was started in standard dose (four doses of $375 \mathrm{mg} / \mathrm{m}^{2}$ with a one-week interval). After the third dose of Rituximab, there was a significant remission of the AIHA and an important improvement of the pulmonary infiltration with a normal chest $\mathrm{x}$ ray image of control in the year 2009 Figure 4A and B.

To present date the patient has received 8 doses of Rituximab. They've been given intravenously every year, no infection complications have been associated during this time, and pulmonary functions did not show any alterations so far.

\section{Discussion}

The present case illustrates the correlation of two distinctive clinical patterns of CVID which

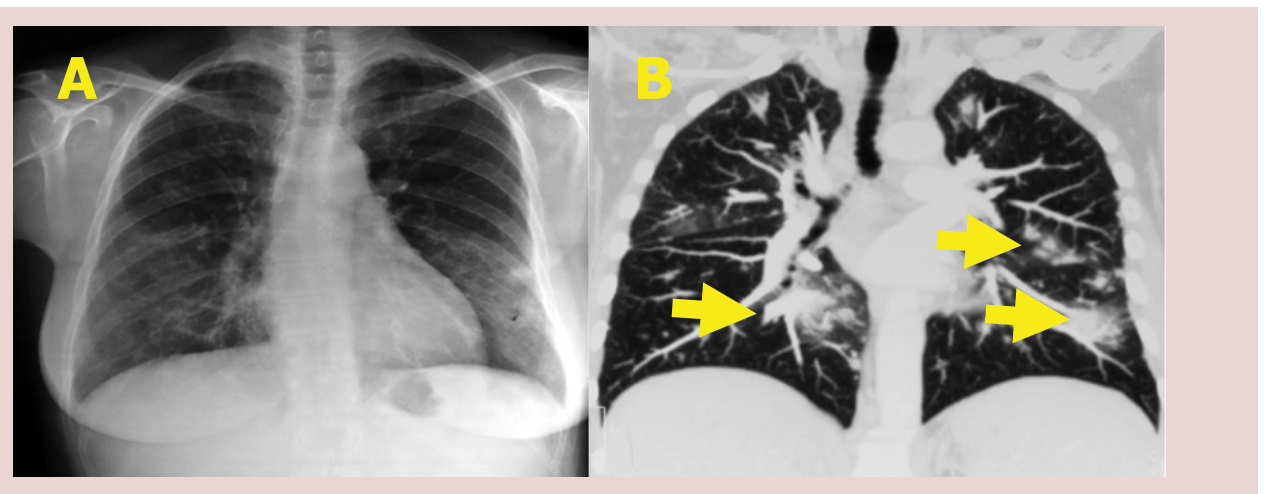

Figure 1. Chest CT and Chest X-ray prior to rituximab. A: Infiltrative disease in left lower lobe, B: Reticulonodular pattern and ground glass images in right lower lobe and left lower lobe (arrows). 


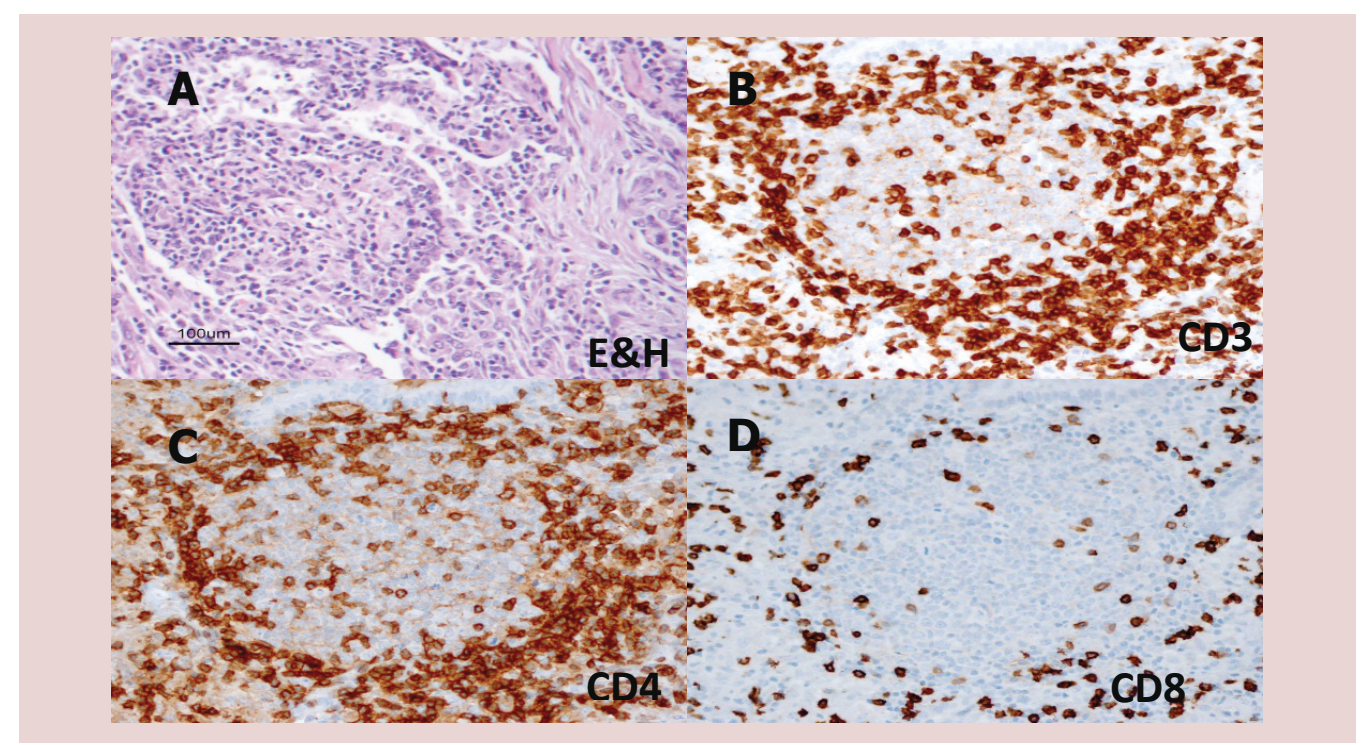

Figure 2. Eosin- hematoxylin stain and immunohistochemistry of the Granulomatous disease present in the patient's lung parenchyma, sample taken from left lower lobe, A: shows a noncaseating granuloma and fibrosis surrounding the granuloma, B: Shows CD3 T cells surrounding the granuloma, C: The majority of T cells are CD4 (helper) cells, D: Shows a minority of CD8 T cells but, nevertheless, they are present.

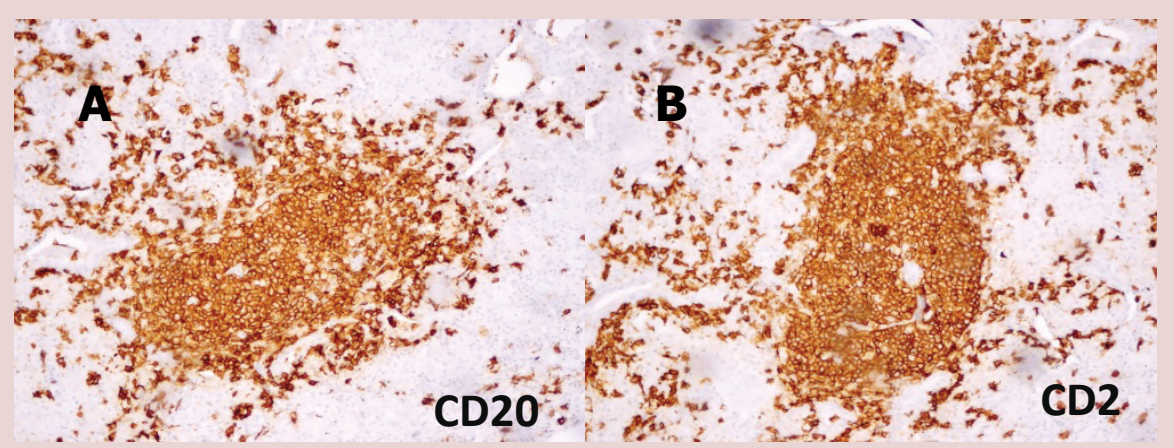

Figure 3. Immunohistochemistry of left lower lobe sample showing two follicular-like aggregates of B-cells only. There were present numerous follicular structures around the granulomas.

\begin{tabular}{|c|c|c|}
\hline Table 1. Functional peripheral blood study & $59 \%$ \\
\hline 1. & Raised effector memory CD4-T-cells (CD4+CD45RA-CCR7) & $24 \%$ \\
\hline 2. & Raised activated CD4-T-cells (CD4+CD38+DR+) & $96 \%$ \\
\hline 3. & Raised activated CD8-T-cells (CD8+CD38+DR+) & $77 \%$ \\
\hline 4. & Raised effector CD8-T-cells (CD8+CD45RA+CCR7-) & $6 \%$ \\
\hline 5. & Low CD4+CD25+ & $1.5 \%$ \\
\hline 6. & Normal CD8+CD25+ & $0.38 \%$ \\
\hline 7. & Low CD4+CD25high & $0.1 \%$ \\
\hline 8. & Very low B-cells (CD19+CD20+) \\
\hline
\end{tabular}

are autoimmune cytopenias and lymphocytic infiltration $[7,8]$. Even though the patient didn't present any clinical manifestation of the pulmonary lesions (with normal examination and normal PFT), her clinical evolution presented only with autoimmune hemolytic anemia and immune thrombocytopenia, this correlation has been described in CVID patients with granulomatous infiltrative disease, and this type of infiltration correlates with higher risk of developing autoimmune processes as manifested in our patient [7]. The lung biopsy showed areas of noncaseating granulomas, majorly composed of CD4+ lymphocytes as shown in Figure 2. Surrounding the granulomas there were areas of only B lymphocytes Figure 3, forming follicular 

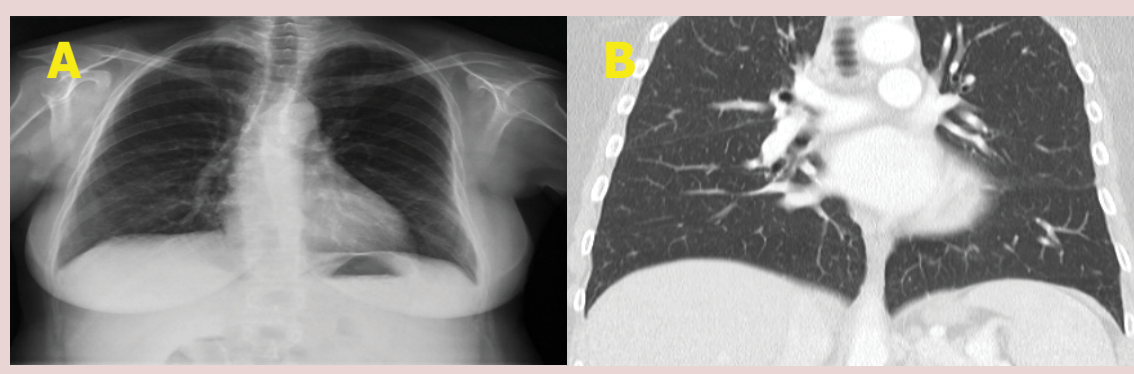

Figure 4. Imaging studies after the use of Rituximab. A: Chest X-ray shows the disappearance of infiltrative pattern in left lower lobe. B: Chest CT scan, showing the disappearance of ground glass images.

like structures. This is a very interesting finding not previously reported and we hypothesize that these B lymphocytes follicle-like formations could be in a way modulating the formation of granulomatous tissue. Also we postulate that the prevention of further reorganization of granulomas and infiltrative lesions in the normal pulmonary tissue could be prevented with the administration of rituximab which will get rid of the infiltrative B lymphocytes. As shown in the pulmonary computed tomography, there was a significant change in the pulmonary lesions, with disappearance of ground glass opacities after 6 months of the initiation of Rituximab. So far, the patient didn't present anymore lesions with a yearly follow up after the initiation of the therapy. Few reports and protocols had been reported with a standard treatment. Most recently a retrospective analysis made by Chase et al. [5], showed that patients with GLILD present an important improvement in both, image parameters and PFT, after the administration of a combined therapy with Rituximab and Azathioprine. In the case of our patient the use of rituximab had the same effect with important resolution of the lesions without the need of another immunosuppressive drug. It is also important to point out that activated $\mathrm{CD} 4$ and $\mathrm{CD} 8 \mathrm{~T}$ cells were raised during the episode of AIHA, but on the other hand, B cells were very low in peripheral blood, and still present in the lung specimen, forming isolated areas surrounding the granulomas. We believe that by depleting the $\mathrm{B}$ cell infiltration in the lungs with Rituximab, there will be less inflammatory signals, thus less granulomatous formation, and the avoidance of fibrosis. But to validate the monotherapy use of Rituximab, clinical trials as monotherapy are required. The maintenance therapy given in between the rituximab sessions included replacement therapy with a $5 \%$ intravenous immunoglobulin preparation at a dose of $400 \mathrm{mg} / \mathrm{kg} /$ month. Immunoglobulin profile done at baseline and following rituximab was as follows: Baseline: IgG $767 \mathrm{mg} / \mathrm{dL}, \operatorname{IgA}<7 \mathrm{mg} / \mathrm{dL}, \operatorname{IgM} 10 \mathrm{mg} /$ dL; after rituximab: IgG $833 \mathrm{mg} / \mathrm{dL}, \mathrm{IgA}<7 \mathrm{mg} /$ $\mathrm{dL}$, IgM $6 \mathrm{mg} / \mathrm{dL}$. The authors were not worried about hypogammaglobulinemia in this patient because at the time of rituximab indication she was on replacement therapy with intravenous immunoglobulin.

\section{Conclusions}

- GLILD is an inflammatory pulmonary complication of CVID that is associated with a worse prognosis and increased prevalence of lymphoproliferative disorders. Disappearance of GLILD after Rituximab suggest a B-cell mediated mechanism in this disease in addition with T-cell abnormalities [1,2,5,9-13].

- Surrounding the granulomatous formations were follicle like structures composed entirely by B lymphocytes. By targeting these cells with rituximab, we believe that further damage was prevented.

- Monotherapy with rituximab for GLILD in CVID, although it seems to be effective as presented in the case, should be further studied to assess its clinical efficacy by clinical trials as monotherapy.

\section{Patient's perspective}

The patient signed the informed consent with the present information translated to English.

"I' $m$ very pleased to know that my case is being reported in the medical biography. Since I started rituximab, I didn't present any more episodes of anemia and so far I hadn't present any complication of my disease. The administration of Rituximab has improved somewhat my 

common variable immunodeficiency. Is single therapy enough?

sensation of tiredness and I have a normal life".

\section{Consent}

"Written informed consent was obtained from the patient for publication of this case report and accompanying images. A copy of the written consent is available for review by the Editor-inChief of this journal."

\section{Competing interests}

"The author(s) declare that they have no competing interests".

\section{Authors' contributions}

Dr. M. Arraya: Wrote the article and followed the evolution of the patient and the treatment, review the data concerning the case, and was a major contributor in writing the manuscript. Dr. J Carbone: administered rituximab and revised date concerning the case as well as assessing the structure of the report. Y. Castro performed the histopathological examination of the pulmonary samples. E. Sarmiento, J. Navarro. And E. FdezCruz, review the laboratory data. All authors read and approved the final manuscript.

\section{References}

1. Bates CA, Ellison MC, Lynch DA et al. Granulomatouslymphocytic lung disease shortens survival in common variable immunodeficiency Clinical Immunology. J. Allergy. Clin. Immunol. 114, 415-21 (2004).

2. Salzer U, Warnatz K, Peter HH. Common variable immunodeficiency an update. Arthritis. Res. Ther. 14, 223 (2012).

3. Park JH, Levinson AI. Granulomatous-lymphocytic interstitial lung disease (GLILD) in common variable immunodeficiency (CVID). Clin. Immunol. 134, 97103 (2010).
4. Mechanic LJ, Dikman S, Cunningham-Rundles C. Granulomatous disease in common variable immunodeficiency. Ann. Intern. Med. 127, 613-7 (1997).

5. Chase NM, Verbsky JW, Hintermeyer MK et al. Use of Combination Chemotherapy for Treatment of Granulomatous and Lymphocytic Interstitial Lung Disease (GLILD) in Patients with Common Variable Immunodeficiency (CVID). J. Clin. Immunol. 33(1), 30-39 (2003).

6. Cunningham-Rundles C, Bodian C. Common variable immunodeficiency, clinical and immunological features of 248 patients. Clin. Immunol. 92, 34-48 (1999).

7. Cunningham-Rundles C. How I treat common variable immune deficiency. Blood. 116, 7-15 (2010).

8. Chapel H, Lucas M, Lee $M$ et al. Common variable immunodeficiency disorders: division into distinct clinical phenotypes. Blood. 112(2), 277-86 (2008).

9. Palanichamy A, Jahn S, Nickles D et al. Rituximab efficiently depletes increased CD20-expressing T-cells in multiple sclerosis patients. J. Immunol. 193, 580-586 (2014).

10. Weiner GJ. Rituximab: Mechanism of Action. Semin. Hematol. 47(2), 115-123 (2010).

11. Hermaszewski RA, Webster AD. Primary hypogammaglobulinemia: a survey of clinical manifestations and complications. Q. J. Med. 86, 31-42 (1993).

12. Kohler PF, Cook RD, Brown WR et al. Common variable hypogammaglobulinemia with $\mathrm{T}$-cell nodular lymphoid interstitial pneumonitis and B-cell nodular lymphoid hyperplasia: different lymphocyte populations with a similar response to prednisone therapy. J. Allergy. Clin. Immunol. 70, 299-305 (1982).

13. Koss MN, Hochholzer L, Langloss JM et al. Lymphoid interstitial pneumonia: clinicopathological and immunopathological findings in 18 Cases. Pathology. 19, 178-185 (1987). 